This item was submitted to Loughborough's Research Repository by the author.

Items in Figshare are protected by copyright, with all rights reserved, unless otherwise indicated.

\title{
Reliability of a novel badminton intermittent exercise protocol
}

PLEASE CITE THE PUBLISHED VERSION

https://doi.org/10.1080/02701367.2019.1620911

PUBLISHER

Taylor and Francis

VERSION

AM (Accepted Manuscript)

PUBLISHER STATEMENT

This is an Accepted Manuscript of an article published by Taylor \& Francis in Research Quarterly for Exercise and Sport on 11 June 2019, available online: http://www.tandfonline.com/10.1080/02701367.2019.1620911.

\section{LICENCE}

CC BY-NC-ND 4.0

\section{REPOSITORY RECORD}

Chia, Jingyi, Jia Yi Chow, Laura Barrett, and Stephen F. Burns. 2019. "Reliability of a Novel Badminton Intermittent Exercise Protocol". figshare. https://hdl.handle.net/2134/38224. 


\section{Research Quarterly for Exercise and Sport}

\section{Reliability of a novel badminton intermittent exercise protocol}

\begin{tabular}{|c|c|}
\hline Journal: & Research Quarterly for Exercise and Sport \\
\hline Manuscript ID & 18-12-PHY-03.R1 \\
\hline Manuscript Type: & Original Article \\
\hline Manuscript Category: & Exercise Physiology \\
\hline Keyword: & Fitness, performance, representative, sport-specific \\
\hline \multicolumn{2}{|c|}{$\begin{array}{l}\text { Note: The following files were submitted by the author for peer review, but cannot be converted to PDF. } \\
\text { You must view these files (e.g. movies) online. }\end{array}$} \\
\hline Supplementary Video 1.mp4 & \\
\hline
\end{tabular}

\section{SCHOLARONE ${ }^{m}$ Manuscripts}


Reliability of a novel badminton intermittent exercise protocol 


\begin{abstract}
Purpose: Badminton is a sport characterized by high-intensity activity interspersed with short rests requiring specific physiological fitness, skills and game tactics. This study aimed to develop, describe and establish the reliability of a novel badminton intermittent exercise protocol, to simulate singles match-play (Part A) and assess match-fitness (Part B). Method: Twelve well-trained male badminton players performed a two-part protocol, mimicking the demands and activity of match-play, on two occasions with at least 72 hours recovery. Part A imitated match-play and consisted of forty stages alternating between $20 \mathrm{~s}$ work - receiving shuttlecocks from a launcher and ghosting (simulated) actions - and $40 \mathrm{~s}$ rest. Part B was an open-ended period, challenging match-fitness, designed to exhaust participants within 10 min. Physiological responses (heart rate, blood lactate and ratings of perceived exertion) (Part A and B) and time to exhaustion (Part B) were determined both times. Results: There were no differences in performance and physiological responses between trials $(p>.05)$. The protocol demonstrated excellent reliability (intraclass correlation coefficient $>0.89$ ) for all variables. Time to exhaustion (Part B) was $198 \pm 98$ s and $194 \pm 82 \mathrm{~s}$, respectively. Mean (Part A) and peak (Part B) heart rates were 166 $\pm 9 ; 191 \pm 8 \mathrm{~b} \cdot \mathrm{min}^{-1}$ and $166 \pm 9 ; 191 \pm 7$ $\mathrm{b} \cdot \mathrm{min}^{-1}$ and blood lactate concentrations (Part B) $11.8 \pm 2.3$ and $11.5 \pm 2.1 \mathrm{mmol} \cdot \mathrm{l}^{-1}$ for trials 1 and 2, respectively. Conclusion: The protocol reliably induces match-like responses and can be used to assess match-fitness for training/research purposes.
\end{abstract}

Keywords: Fitness, performance, representative, singles, sport-specific 
A typical badminton men's singles match lasts for an average of 40-60 minutes with a workto-rest ratio of 1:2 (Gawin, Beyer, \& Seidler, 2015; Phomsoupha \& Laffaye, 2015). The intermittent and high intensity activity pattern of the game places considerable demands on both the aerobic and anaerobic energy systems of players (Faude et al., 2007). It is therefore important that badminton players are able to resist fatigue and sustain this repeated highintensity work for the entire duration of the match for successful performances (Faude et al., 2007; Madsen, Hojlyng, \& Nybo, 2016).

While changes in badminton performance with successive matches has been studied during actual match-play (Abian-Vicen et al., 2014; Abián-Vicén, Del Coso, González-Millán, Salinero, \& Abián, 2012), the variability inherent to each match makes it difficult to translate findings from one study to another. Moreover, measuring the ability of a player to last in a match scenario is desirable both for the player for their own development and for coaches to design appropriate training interventions. However, unlike running or cycling where performance can be easily measured using a time trial or time to exhaustion test, the dynamic nature of badminton makes this difficult to achieve. To overcome the variability present in matches, standardized badminton specific protocols have been developed to replicate the demands of actual match-play and can be considered as a more controlled alternative for examining outcomes for research purposes (Chin et al., 1995; Fuchs, Faude, Wegmann, \& Meyer, 2014; Madsen et al., 2016; Wonisch, Hofmann, Schwaberger, von Duvillard, \& Klein, 2003). The strength of the findings of these studies, however, are largely dependent on how realistic the exercise protocol is and its ability to mimic actual match-play.

Key features of existing protocols involve the use of specific footwork techniques and on-court movements conducted in specified sequences and directions as indicated by light flashes of 
increasing speeds in three-minute work stages (Chin et al., 1995; Fuchs et al., 2014). Unlike other protocols, the B-ENDURANCE test by Madsen and colleagues (2016) utilizes a randomized sequence and work periods ranging from 18 to 30 seconds, to simulate the movement and timing patterns of match-play. While the required court movements are effectively captured in these protocols, most of the work-to-rest periods $(\sim 3: 1)$ are largely exaggerated in comparison to the typical 1:2 ratio (Chin et al., 1995; Fuchs et al., 2014; Madsen et al., 2016; Phomsoupha \& Laffaye, 2015).

Secondly, as intensity of match-play does not increase with duration in badminton, the inclusion of incremental levels of intensity is likely to limit performance and may therefore be more suited for determining maximal aerobic capacity or cardiorespiratory parameters at specific time points (e.g. respiratory compensation point) rather than simulating actual matchplay (Brechbuhl, Girard, Millet, \& Schmitt, 2016). A further limitation of extant protocols is the failure to include the actual return of the shuttlecock which limits the representativeness of the tests (Ferrauti, Kinner, \& Fernandez-Fernandez, 2011). Shot frequency during a men's singles match is approximately one shuttle per second (Phomsoupha \& Laffaye, 2015). As such, a considerable level of arm power and endurance is needed to consistently produce powerful strokes throughout the match, contributing to the level of fatigue typically experienced by players. Recent development of portable shuttle feeders/launchers and badminton robots such as Baddy ${ }^{\odot}$ (Baddy, 2018), Knight Trainer ${ }^{\odot}$ (Knight, 2018), and Siboas $^{\odot}$ (Siboasi, n.d) might therefore provide a viable tool to enhance the representativeness of these protocols.

Finally, movements during a match are dependent on player-player interactions arising from the different shot types (e.g. clear, drop) used. Hence, a deliberate inclusion of realistic rallies 
is needed to reflect match behaviour. A representative task design ensures that the individual utilizes the same informational sources during the exercise protocol to organize their actions as they would in an actual scenario (Chow, Davids, Button, \& Renshaw, 2016), allowing the results to be extended to understanding performance in a real-game context. Hence, any protocol used should mimic the activity and patterns of movement of the game as much as possible and importantly, the return of shots cannot be neglected.

Thus, the aim of this study was to develop and assess the reliability of a novel badminton intermittent exercise protocol. The protocol incorporates movement patterns and shots to form realistic rally sequences together with the need for players to return actual shots, thereby closely simulating the demands and activity profile of actual match-play. The protocol was designed in two-parts - Part A is a systematic 40-min protocol to mimic badminton match-play and Part B, provides a fatiguing open-ended protocol which can be extended after Part A to assess match fitness.

\section{Method}

The study was designed and conducted to develop a more ecologically valid badmintonspecific exercise protocol and evaluate its reliability and whether it provides a good physiological simulation of actual match play. A repeated-measures study design was used to determine the reliability and physiological responses - heart rate (HR) and blood lactate concentration $(\mathrm{BLa})$ were measured and compared against data from the existing literature.

\section{Participants}

Twelve well-trained male badminton players (mean \pm SD: age $22.8 \pm 1.9$ years, body mass $67.3 \pm 8.8 \mathrm{~kg}$, height $1.7 \pm 0.7 \mathrm{~m}$ ) participated in this study. The players averaged $11.3 \pm 2.4$ 
years of competitive playing experience, having a wide range of representation from college to international level. They were training actively for an average of $5 \pm 2 \mathrm{~h}$ per week. Participation was voluntary and written informed consent was obtained prior to testing. The study was approved by the University Institutional Review Board and adhered to the guidelines for ethical practice [IRB-2015-05-009].

\section{Badminton intermittent exercise protocol - Design, Development and Procedures}

The design of the badminton intermittent exercise protocol was based on an extensive examination of observational and notational scientific literature on badminton matches and pilot experimentation by the researchers with the aid of a male badminton player with 14 years of competitive playing experience - the highest being collegiate level and local club competitions. The design of the protocol involved finding the best combination of rally sequences based on the following factors of consideration: (a) rally sequences and shot frequencies which mimic actual match-play, (b) realistic distribution of shot types, (c) intermittent activity period; alternating between work and rest and, (d) eliciting match-like physiological responses.

As previous protocols involved mainly badminton footwork without any shuttles fed to them (Chin et al., 1995; Fuchs et al., 2014; Madsen et al., 2016; Wonisch et al., 2003), the use of random sequences in these protocols did not pose much of an issue (e.g. participants simply had to get to the specified court location within the stipulated time). However, with the incorporation of actual shots, a random sequence would neither be logical nor realistic. For instance, it is unlikely that a smash shot is used to return a drop shot during a match. Therefore, the sequences were deliberately designed to simulate match-play conditions as closely as 
possible. Moreover, to elicit match-like fatigue and physiological responses, the number of shots per sequence and shot types need to be similar to actual match-play data.

Subjects were tested on the protocol on two separate occasions, separated by at least $72 \mathrm{~h}$ to allow for complete recovery while maintaining the individual's fitness level. To account for any time-of-day effects, the second session was scheduled with a time-of-day difference of no more than one hour from the first. Subjects were instructed to refrain from any physical activity $24 \mathrm{~h}$ before each session. On the test day, they reported to the court and were fitted with HR monitors (Polar RCX5 Heart Rate Monitor, Polar Electro, Kempele, Finland). Subjects were given 15 min to warm-up as they would prior to a match and performed the first level of the protocol for familiarization. They repeated the same warm-up for both sessions. The actual protocol consists of two parts - Part A and Part B.

\section{Part A is a fixed duration of 40 one-minute stages. For each stage, subjects work for $20 \mathrm{~s}$,}

followed by $40 \mathrm{~s}$ of rest. The exercise protocol required subjects to perform court movements and shot types at a work-to-rest ratio of 1:2 to mimic the activity patterns during an actual match. This involves returning shuttles launched by a feeding machine (SS-YS-8000, Siboasi, China) to different points on the court as well as ghosting actions. The accuracy of the feeds from the launcher was assessed by launching 30 new shuttlecocks (Li-Ning Grandpix Gold Speed 77) towards a $0.5 \mathrm{~m}^{2}$ target box. Six trials of 30 shuttles (180 shuttles in total) were performed for each of four extreme corners of one half of the badminton court. A chi-square test was performed, and no significant differences were observed between the four locations $\left(\chi^{2}(3)=0.305, p=0.959\right)$, illustrating the precision of the launcher across points. In terms of accuracy, the machine fed shuttles which landed in the target boxes $>89 \%$ of the time in all four corners of the court $(89.4-91.1 \%)$. Given the lack of existing data to compare the 
accuracy of the launcher, these values were compared to serve accuracy measured in badminton players whereby the smallest percentage of missed serves was approximately 30 \% (Clarke \& Duncan, 2015; Edwards, Lindsay, \& Waterhouse, 2005). The greater accuracy of the launcher further validates its use in this study as opposed to a human to reduce the possible variance within the test. The use of a launcher was relevant in this case to eliminate any fatigue-related deviations in feeds, ensuring consistent and standardized feeds to all participants for both trials even though the use of a "live receiver" would have further increased the level of task representativeness. The test continues even on instances where the launcher fails to launch accurately.

To launch the different shot types to the desired points in a realistic manner, requires the shifting of the launcher. For instance, it is unlikely that an overhead clear shot is played from the net area. The various positions of the launcher and markers demarcating the ghosting actions during the protocol and associated instructions for the guiding markers are illustrated in Figure 1.

***Insert Figure 1 about here***

During the protocol, participants used badminton-specific footwork movements (e.g. lunges, chasse steps) and shot types as per an actual match. The distribution of shot types used is presented in Table 1 and was determined based on previous time-motion and notational analyses (Chee, Chen, \& Asok, 2008; Lee, Xie, \& Teh, 2005; Phomsoupha \& Laffaye, 2015) with the smash and net shots being the two most frequent shot types.

\footnotetext{
***Insert Table 1 about here***
} 
The work periods for each stage consist of specific drill sequences; alternating between returning the shuttles fed from the launcher and performing ghosting actions to simulate specific shot types. Average number of shots for each work period was six actual feeds and six ghosting actions. An example sequence is illustrated in Figure 2 and Supplementary Video 1 - Diagonal.

***Insert Figure 2 about here***

Here, the subject returns a net shot and goes to the back of the court for a drop shot action before going forward again to return the next net shot. This mimics the playing pattern of a match whereby it is common for a net shot to follow a drop shot or vice versa to tire the opponent and potentially win the point (McGarry, 2013). Each sequence is performed four times; twice diagonally and twice in a straight manner. The entire set of sequences $(n=10)$ is performed diagonally first before straight; Sequence 1-10 diagonally before going back to Sequence 1 in a straight manner. During the rest period before the next sequence type, the participant was shown a video outlining the drill and the experimenter also provided verbal reminders on the respective court positions and shot(s) to perform. Once the first shuttle is launched, the subject will know which side the next shuttle would be launched as the launcher works in left-right manner.

Part B is an open-ended period consisting of one-minute stages, divided between $40 \mathrm{~s}$ work and $20 \mathrm{~s}$ rest, designed to exhaust the participant (Nicholas, Nuttall, \& Williams, 2000). Similar to Part A, each stage lasts for one minute. However, the participants are required to work for $40 \mathrm{~s}$, followed by $20 \mathrm{~s}$ of rest. The drill sequence (return a smash shot followed by a 
dummy push shot with one foot on the service line) is repeated every minute at the same intensity until the participant reaches voluntary exhaustion or when he misses two shuttles in a row. Despite the same drill sequence in Part B, it is unlikely that performance would be affected by boredom as this part was designed to exhaust participants in a short period of time ( $\leq 10 \mathrm{~min}$ ) (Atkinson \& Nevill, 2005; Nicholas et al., 2000). The average number of shots for each stage was 12 actual feeds and 12 ghosting actions. The full set of sequences can be found in the Supplementary Table 1.

The HR was recorded continuously throughout both parts of the protocol at 5-second intervals. A fingertip blood sample for measurement of blood lactate was taken from the nonplaying arm in a sitting position using a hand-held Lactate Pro Analyzer (Arkray, Tokyo, Japan) immediately at the point of termination in Part B of the protocol. Subjective ratings of perceived exertion (RPE) were also recorded after every work period (Part A) and immediately at the point of termination (Part B) from a scale of 6 - 20 (Borg, 1973).

\section{Statistical analysis}

All data are presented as their mean values and SDs. Normality of data was examined using the Shapiro-Wilk test (Field, 2009). A selection of statistical methods was used to determine the repeatability and agreement between the two trials for all measured variables. To examine test-retest reliability, pairwise comparisons were first applied to determine any possible learning effects using a paired t-test. Relative reliability was expressed in terms of intraclass correlation coefficient (ICC) and $95 \%$ confidence limits were determined for all the ICCs (Hopkins, 2000). An ICC $>0.75=$ excellent reliability, $0.4-0.75=$ fair to good reliability and $<0.4=$ poor reliability (Fleiss, 1981). Absolute reliability was determined using limits of agreement (LOA) as examined by Bland Altman plots and expressed in terms of typical error 
of measurement (TEM) and coefficient of variation ( \% CV) (Atkinson \& Nevill, 1998). The calculated CV represents the mean of the within participant variation. The level of statistical significance was set at $p<.05$. Apart from the Bland Altman and CV calculations which were performed using GraphPad Prism version 7.0 and Hopkins reliability spreadsheets respectively, all other statistical analyses were performed using SPSS version 24.0.

\section{Results}

For both trials, the protocol was terminated with all participants reaching voluntary exhaustion. A summary of the data and reliability statistics for all performance and physiological variables during both trials, including the ICC, TEM, $95 \%$ LOA and CV values are shown in Table 2.

***Insert Table 2 about here***

Based on the ICCs, excellent test-retest reliability $(>0.80)$ was observed for all variables. Physiological responses (HR, blood lactate concentration, RPE) demonstrated a CV of 0.3 $5.8 \%$. There were no significant differences in the number of stages completed nor time to exhaustion, suggesting no significant test-retest bias. The number of stages completed in Part B was $4 \pm 2$ and $4 \pm 1$ (TEM: 0.5; CV: $13.0 \%$ ) and time to exhaustion was $198 \pm 98 \mathrm{~s}$ and $194 \pm 82$ s (TEM: 27.9; CV: $14.7 \%$ ) for Trials 1 and 2 respectively (Figure 3). The respective Bland Altman plots for performance are presented in Figure 4. The plots show one individual whose performance was out of the $95 \%$ LOA.

***Insert Figure 3 and 4 about here*** 
There were also no significant differences in both HR responses and BLa between trials ( $p>$ 0.05) (Figure 5). Mean $\left(166 \mathrm{~b} \cdot \mathrm{min}^{-1}\right)$ and peak $\left(191 \mathrm{~b} \cdot \mathrm{min}^{-1}\right)$ HR were the same for Trials 1 and 2. Likewise, there was no significant difference in mean BLa between trials (Trial 1: 11.8 $\pm 2.3 \mathrm{mmol} \cdot \mathrm{l}^{-1}$; Trial 2: $\left.11.5 \pm 2.1 \mathrm{mmol} \cdot \mathrm{l}^{-1}\right)$. In terms of subjective responses, participants reported similar RPE at the point of exhaustion for Trial $1(19 \pm 1)$ and $2(20 \pm 1)$, translating to "extremely hard to maximal exertion" based on Borg's (1973) 6-20 scale.

***Insert Figure 5 about here***

\section{Discussion}

The aim of this study was to assess the reliability of a novel badminton intermittent exercise protocol, designed to mimic actual match-play as closely as possible through realistic footwork patterns combined with incorporating the actual returns of shots and realistic shot type distributions. The protocol demonstrated good repeatability between trials with ICC values $>0.89$ for all performance and objective markers of physiological stress (HR and BLa). In addition, the physiological responses observed were comparable to those measured during actual match-play (Cabello, Padial, Lees, \& Rivas, 2004; Faude et al., 2007; Manrique \& González-Badillo, 2003); suggesting that the protocol accurately reflects the demands of a badminton singles match and has potential to be used in future research studies.

Apart from the performance measures (CV: $13.0 \%), \mathrm{CV}$ values for all variables were small $(<6.0 \%)$. The higher $\mathrm{CV}$ for performance measures may be attributed to individual differences in fitness status and/or the wide range in level of play as evidenced by the range in stages completed ( $2-6$ stages $)$ and time to exhaustion $(90-340 \mathrm{~s})$ among players for Part $\mathrm{B}$ of the protocol. However, time to exhaustion protocols in general typically have larger CV 
values (>10\%) as compared to time trials (Currell \& Jeukendrup, 2008). This may be explained by the relationship between exercise duration and power output given that small changes in one's power output can lead to large changes in time to exhaustion (Hopkins, Schabort, \& Hawley, 2001). Therefore, the interpretation of CV values must be done with care, depending on whether time to exhaustion or time trial results are more meaningful in the given situation.

While the CV for the B-ENDURANCE protocol is lower (7.9 \%) (Madsen et al., 2016), the lack of representativeness limits its usefulness for experimental purposes. The BENDURANCE consists of randomized movement patterns to four corners of the court which were proposed to mimic match-play with the stochastic movements in random directions. This however, is not representative of the activity pattern of actual match-play whereby the action and reaction of players are interdependent (Walter, Lames, \& McGarry, 2007). This coupling of movement between players can be explained by the dynamical systems theory which posits that coordinated behaviour is a result of the interaction between various factors including player-player relationships (Chow, Seifert, Hérault, Chia, \& Lee, 2014; McGarry, Anderson, Wallace, Hughes, \& Franks, 2002). When applied in the game of badminton singles, players aim to defend a central position by returning to it after every shot as it allows them to reach all four corners of the court equally fast (Wadood, 2014). Likewise, players also try to force their opponent away from this central position by using different shot types, resulting in a "synchronized" activity pattern (Chow et al., 2014). Together with the absence of the need to return shots, the lack of information on physiological measures make it hard to ascertain if the protocol elicited similar responses to an actual match (Kilit, Senel, Arslan, \& Can, 2016). 
The protocol in the present study has overcome these limitations with the inclusion of realistic rally sequences and shot returns. It is also representative of the actual movement pattern as players are required to cover the entire court using court-specific movements. Furthermore, the protocol is the first to consist of comparable proportions of the shot types used during an actual singles match with the smash (current $16.4 \%$ vs. $13.0 \%$ ), net (current $16.1 \%$ vs. $17.0 \%$ ) and lift/lob (current $14.2 \%$ vs. $22.0 \%$ ) shots making up three of the highest percentage of all shots used (Lee et al., 2005; Phomsoupha \& Laffaye, 2015). Players are also working at a 1:2 work-to-rest ratio which closely mimics the activity pattern of an actual match. The specificity of the protocol thus greatly enhances its external validity, usefulness and applicability as compared to existing badminton protocols.

Compared with previous notational analyses in badminton, both mean and peak HR values coincided with that measured during actual match-play. Specifically, mean HR during both trials was $166 \mathrm{~b} \cdot \mathrm{min}^{-1}$, falling in the range of $157-179 \mathrm{~b} \cdot \mathrm{min}^{-1}$ reported in the existing literature. Similarly, peak HR recorded in this study $\left(191 \mathrm{~b} \cdot \mathrm{min}^{-1}\right)$ agreed with the values reported in a recent review by Phomsoupha and Laffaye (2015). In terms of blood lactate concentration, individual blood lactate concentration at the point of exhaustion (7.5 to 14.8 $\mathrm{mmol} \cdot \mathrm{r}^{-1}$ ) and mean concentrations for Trials 1 and 2 were $11.8 \pm 2.3 \mathrm{mmol} \cdot \mathrm{l}^{-1}$ and $11.5 \pm 2.1$ mmol $\cdot 1^{-1}$. While these values fall within the range of $1.9 \pm 12.2 \mathrm{mmol} \cdot \mathrm{l}^{-1}$ documented in the literature, the large variability owing to differences in time of measurement, study design as well as age, and/or training status of the participants makes it difficult to compare. None of the previous studies have measured blood lactate at the point of exhaustion in a match scenario. Instead, blood lactate concentration was often measured during match-breaks, at the end of a match (not point of exhaustion) and post-match recovery periods (Cabello et al., 2004; Deka, 2010; Faude et al., 2007) due to the inherent difficulty in doing so. In addition, 
the values measured in this study compared well against the criteria of blood lactate concentration being above $8.0 \mathrm{mmol} \cdot \mathrm{l}^{-1}$ for reaching maximal oxygen uptake $\left(\dot{\mathrm{V}} \mathrm{O}_{2 \max }\right)$ (Howley, Bassett, \& Welch, 1995). These values also meet the stricter criteria of $9.0 \mathrm{mmol} \cdot \mathrm{l}^{-1}$ recommended by Edvardsen and colleagues (2014) specifically for males aged 20 to 49 years.

Collectively, these results provide strong evidence of the test-retest reliability and physiological validity of this badminton intermittent exercise protocol. While it is important to note that the $\mathrm{CV}$ values of the performance measures were above $10 \%$, this is also common in many time to exhaustion studies (Currell \& Jeukendrup, 2008). Due to the lack of a gold standard badminton exercise test and the lack of representativeness of a standard $\dot{V}$ $\mathrm{O}_{2 \max }$ test, it is difficult to compare the results of the protocol against any established standards. However, it would be expected that better and higher ranked badminton players would perform better during this protocol. With this purpose in mind, eight elite male badminton players performed the exercise protocol to voluntary exhaustion as part of a separate unpublished study. All participants had competed at international level during the previous year and were considered to be of a higher skill level as compared to those in this study. Results from an independent t-test showed that the mean time to exhaustion for Part B of the exercise protocol was significantly longer for the elite players $(304 \pm 95 \mathrm{~s})$ as compared to the participants in this study $(196 \pm 89 \mathrm{~s})(\mathrm{t}(18)=2.613, p=0.018)$ (unpublished data). However, within the elite players there was no correlation with World Rankings at the time of testing and time to exhaustion $\left(\mathrm{R}_{\mathrm{s}}=0.26, p>.05\right)$. This may suggest that exercise duration for Part B of the protocol is not simply reflective of greater skill level but that match-fitness may be an important contribution to exhaustion time. Moreover, given that time to exhaustion was less than 10 minutes, it is unlikely that the players stopped due to boredom or the need to fulfil other obligations as highlighted by Atkinson and Nevill (2005). This 
highlights the potential of using this protocol for coaches to distinguish players of different levels of match fitness in future studies.

One of the limitations of this protocol is that while the launcher was used to ensure the feeds were consistent and controlled, this affected the actual perception-action coupling where a player needs to accurately perceive their opponent's body language and court position during a match. Nonetheless, this protocol has the potential to be used to induce match-like demands and fatigue levels in a more standardized and controlled manner in a research setting. For instance, future studies can investigate the effectiveness of supplementation or training intervention in a pre-post design using this protocol which eliminates the variability present if an actual match was played for each session of the research study. In addition, given the intermittent profile of the protocol, coaches and practitioners may consider using it as a match-fitness monitoring tool as opposed to using the traditional multistage 20 -metre shuttle run test or existing badminton specific endurance protocols which lack task representativeness. Additional considerations include the cost of purchasing the launcher and its ease of use. While there are several launchers which can perform the shots necessary for this protocol, they differ in terms of their size, ease of programming and therefore, cost. In summary, this protocol induced match-like physiological responses in badminton singles players. Test-retest reliability was also high but further research of the application of this protocol in distinguishing skill level is warranted. It may also be beneficial to include a familiarization trial to reduce the variability observed and minimize any potential learning effect(s).

\section{What does this article add?}


Ecologically valid representative tasks are important tools for coaches and athletes in assessing performance and training improvements. For the game of badminton, traditional multistage 20-metre shuttle run test or existing badminton specific endurance protocols which lack task representativeness are typically administered to determine a player's fitness level. Considering the activity profile of badminton, there is a need for more match-specific designs of exercise protocols rather than pure aerobic or anaerobic regimes. This enhances the relationship between information and action by simulating conditions of competitive performance to encourage greater accuracy of data and specific transfer of learning.

This study was the first ecologically valid protocol that not only incorporates realistic intermittent profile and rally sequences but more importantly, considers the physical and muscular demands of hitting the shuttle as opposed to simply performing the stroke action. The protocol would therefore prove useful to athletes and coaches who want to regularly assess or monitor changes in match-fitness. Furthermore, the excellent reliability demonstrated also opens up opportunities for potential application for researchers who wish to examine the effects of supplementation and/or other interventions for enhancing badminton performance. Improved badminton performance may potentially result from this protocol as the ability to sustain high-intensity rallies may allow a player more "flexibility" in tactical strategy. 


\section{References}

Abian-Vicen, J., Castanedo, A., Abian, P., Gonzalez-Millan, C., Salinero, J. J., \& Del Coso, J. (2014). Influence of successive badminton matches on muscle strength, power, and body-fluid balance in elite players. International Journal of Sports Physiology and Performance, 9(4), 689-694. doi: 10.1123/ijspp.2013-0269

Abián-Vicén, J., Del Coso, J., González-Millán, C., Salinero, J. J., \& Abián, P. (2012). Analysis of dehydration and strength in elite badminton players. PLoS One, 7(5), 1-8. doi: 10.1371/journal.pone.0037821

Atkinson, G., \& Nevill, A. (2005). Mathematical constants that vary? Medicine and Science in Sports and Exercise, 37(10), 1822; author reply 1823.

Atkinson, G., \& Nevill, A. M. (1998). Statistical methods for assessing measurement error (reliability) in variables relevant to sports medicine. Sports Medicine, 26(4), 217-238. doi: 0112-1642/98/0010-0217/\$11.00/0

Baddy. (2018). Open source badminton robot. from https://www.baddy.space/

Borg, G. A. (1973). Perceived exertion: A note on "history" and methods. Medicine and Science in Sports, 5(2), 90-93.

Brechbuhl, C., Girard, O., Millet, G. P., \& Schmitt, L. (2016). On the use of a test to exhaustion specific to tennis (TEST) with ball hitting by elite players. PLoS One, 11(4), e0152389. doi: 10.1371/journal.pone.0152389

Cabello, D., Padial, P., Lees, A., \& Rivas, F. (2004). Temporal and physiological characteristics of elite women's and men's singles badminton. International Journal of Applied Sports Sciences, 16(2), 1-12.

Chee, L., M, , Chen, C., K , \& Asok, K., G. (2008). Time motion and notational analysis of 21 point and 15 point badminton match play. International Journal of Sports Science and Engineering, 2(4), 216-222. 
Chin, M. K., Wong, A. S., So, R. C., Siu, O. T., Steininger, K., \& Lo, D. T. (1995). Sport specific fitness testing of elite badminton players. British Journal of Sports Medicine, 29(3), 153-157.

Chow, J. Y., Davids, K., Button, C., \& Renshaw, I. (2016). Nonlinear pedagogy in skill acquisition: An introduction: Routledge.

Chow, J. Y., Seifert, L., Hérault, R., Chia, S. J. Y., \& Lee, M. C. Y. (2014). A dynamical system perspective to understanding badminton singles game play. Human Movement Science, 33, 70-84. doi: http://dx.doi.org/10.1016/j.humov.2013.07.016

Clarke, N. D., \& Duncan, M. J. (2015). Effect of carbohydrate and caffeine ingestion on badminton performance. International Journal of Sports Physiology and Performance. doi: 10.1123/ijspp.2014-0426

Currell, K., \& Jeukendrup, A. E. (2008). Validity, reliability and sensitivity of measures of sporting performance. Sports Medicine, 38(4), 297-316. doi: 10.2165/00007256200838040-00003

Deka, P. K. (2010). Physiological responses of recreational badminton match play. (Master of Science), University of Nebraska. (1479189)

Edvardsen, E., Hem, E., \& Anderssen, S. A. (2014). End criteria for reaching maximal oxygen uptake must be strict and adjusted to sex and age: A cross-sectional study. PLoS One, 9(1), e85276. doi: 10.1371/journal.pone.0085276

Edwards, B. J., Lindsay, K., \& Waterhouse, J. (2005). Effect of time of day on the accuracy and consistency of the badminton serve. Ergonomics, 48(11-14), 1488-1498. doi: $10.1080 / 00140130500100975$

Faude, O., Meyer, T., Rosenberger, F., Fries, M., Huber, G., \& Kindermann, W. (2007). Physiological characteristics of badminton match play. European Journal of Applied Physiology, 100(4), 479-485. doi: 10.1007/s00421-007-0441-8 
Ferrauti, A., Kinner, V., \& Fernandez-Fernandez, J. (2011). The Hit \& Turn Tennis Test: An acoustically controlled endurance test for tennis players. Journal of Sports Sciences, $29(5), 485-494$.

Field, A. (2009). Discovering statistics using SPSS. Thousand Oaks: SAGE

Fleiss, J. L. (1981). Statistical methods for rates and proportions. New York: John Wiley.

Fuchs, M., Faude, O., Wegmann, M., \& Meyer, T. (2014). Critical evaluation of a badminton-specific endurance test. International Journal of Sports Physiology and Performance, 9(2), 249-255. doi: 10.1123/ijspp.2012-0387

Gawin, W., Beyer, C., \& Seidler, M. (2015). A competition analysis of the single and double disciplines in world-class badminton. International Journal of Performance Analysis in Sport, 15(3), 997-1006.

Hopkins, W. G. (2000). Measures of reliability in sports medicine and science. Sports Medicine, 30(1), 1-15.

Hopkins, W. G., Schabort, E. J., \& Hawley, J. A. (2001). Reliability of power in physical performance tests. Sports Medicine, 31(3), 211-234.

Howley, E. T., Bassett, D. R., Jr., \& Welch, H. G. (1995). Criteria for maximal oxygen uptake: Review and commentary. Medicine and Science in Sports and Exercise, 27(9), 1292-1301.

Kilit, B., Senel, O., Arslan, E., \& Can, S. (2016). Physiological responses and match characteristics in professional tennis players during a one-hour simulated tennis match. Journal of Human Kinetics, 51, 83-92. doi: 10.1515/hukin-2015-0173

Knight, B. (2018). The Knight Trainer. from http://www.knighttrainer.com/knight_trainer.php 
Lee, K. T., Xie, W., \& Teh, K. C. (2005). Notational analysis of international badminton competitions. Paper presented at the International Society of Biomechanics in Sports, Beijing, China.

Madsen, C. M., Hojlyng, M., \& Nybo, L. (2016). Testing of badminton specific endurance. Journal of Strength and Conditioning Research, 30(9), 2582-2590. doi: 10.1519/jsc.0000000000001350

Manrique, D. C., \& González-Badillo, J. J. (2003). Analysis of the characteristics of competitive badminton. British Journal of Sports Medicine, 37(1), 62.

McGarry, T. (2013). Sport competition as a dynamical self-organizing system: Coupled oscillator dynamics of players and teams underscores game rhythm behaviours of different sports. In Tim McGarry, Peter O'Donoghue \& J. Sampaio (Eds.), Routledge Handbook of Sports Performance Analysis: Routledge.

McGarry, T., Anderson, D. I., Wallace, S. A., Hughes, M. D., \& Franks, I. M. (2002). Sport competition as a dynamical self-organizing system. Journal of Sports Sciences, 20(10), 771-781. doi: 10.1080/026404102320675620

Nicholas, C. W., Nuttall, F. E., \& Williams, C. (2000). The Loughborough Intermittent Shuttle Test: A field test that simulates the activity pattern of soccer. Journal of Sports Sciences, 18(2), 97-104. doi: 10.1080/026404100365162

Phomsoupha, M., \& Laffaye, G. (2015). The science of badminton: Game characteristics, anthropometry, physiology, visual fitness and biomechanics. Sports Medicine, 45(4), 473-495. doi: 10.1007/s40279-014-0287-2

Siboasi. (n.d). Badminton machine. from http://www.siboasiballmachine.com/ball-machine1/siboasi-high-end-auto-shuttlecock-launcher.html

Wadood, T. (2014). Badminton essentials. Oslo, Norway: Total Health. 
Walter, F., Lames, M., \& McGarry, T. (2007). Analysis of sports performance as a dynamical system by means of the relative phase. International Journal of Computer Science in Sport, 6(2), 35-41.

Wonisch, M., Hofmann, P., Schwaberger, G., von Duvillard, S. P., \& Klein, W. (2003). Validation of a field test for the non-invasive determination of badminton specific aerobic performance. British Journal of Sports Medicine, 37(2), 115-118. 


\section{Figure captions}

Figure 1. (a) Front-wheel positions of the machine, (b) markers used for ghosting purposes in the protocol and (c) instructions to the participant for the markers.

Figure 2. Overview of the experimental set-up illustrating a sample sequence of the protocol. Figure 3. Test-retest reliability of (a) the number of stages completed and (b) time to exhaustion $(n=12)$. Dashed line is the identity line $(x=y)$. Blue and red circles indicate a duplicate of two and three participants respectively.

Figure 4. Bland-Altman plot for test-retest scores for (a) number of stages completed and (b) time to exhaustion $(n=12)$. Blue and red circles indicate a duplicate of two and three participants respectively.

Figure 5. Test-retest reliability of (a) mean heart rate, (b) peak heart rate, and (c) blood lactate response $(n=12)$. Dashed line is the identity line $(x=y)$. 


\section{Responses to reviewers' comments.}

We would like to thank the Reviewers and Editor for their careful review of our manuscript and constructive feedback which proved very helpful. We have tried to respond thoroughly and to the best of our ability and believe that the revised version is much improved. Below is our itemized response. $\mathbf{C}$ stands for Comment, $\mathbf{R}$ for Response.

\section{$\underline{\text { Responses to Reviewer } 1 \text { (Highlighted in yellow) }}$}

C1. This sentence might change like this. Time to exhaustion and physiological responses such as heart rate, blood lactate and ratings of perceived exertion were measured for both trials.

R1. Thank you for your suggestion. We have modified the abstract heavily based on some Comments from Reviewer 2 but in keeping with the Journal word limit. The revised sentence reads, "Physiological responses (heart rate, blood lactate and ratings of perceived exertion) (Part A and B) and time to exhaustion (Part B) were determined both times." We hope this is acceptable with the Reviewer and improves the clarity of the sentence.

\section{C2. Concentrations}

R2. We have made the requested change.

C3. It should add this article to support this sentence - Ferrauti, A., Kinner, V., \& FernandezFernandez, J. (2011). The Hit \& Turn Tennis Test: an acoustically controlled endurance test for tennis players. Journal of sports sciences, 29(5), 485-494.

R3. Thank you for directing us to this relevant reference. We have now included the suggested reference to support the importance of including the actual return of the shuttlecock to enhance the representativeness of the tests.

\section{C4. BLa}

R4. We have made the requested change. 


\section{C5. Not necessary}

R5. We have deleted the age details as requested. The phase "an experienced" has also been removed to improve the structure and flow of the sentence.

\section{C6. Add code or number of ethical procedure}

R6. We have included the details as requested.

\section{C7. HR}

R7. We have made the requested change.

\section{C8. How many seconds interval or beat to beat?}

R8. We have clarified the sentence and have included the phrase "at 5-second intervals".

\section{C9. As soon as possible after the sets?}

R9. We have clarified the sentence and have included the phrase "immediately" before the phase "at the point of termination".

\section{C10. When? Like BLa?}

R10. We have clarified the sentence and have included the phrase "after every work period and immediately at the point of termination".

\section{C11. Physiological responses}

R11. The phrase "Objective and subjective variables" has been replaced with "Physiological responses" as requested.

\section{C12. BLa}

R12. We have made the requested change. 


\section{C13. BLa}

R13. Same as above.

\section{C14. HR and BLa}

R14. The phrase "blood lactate concentration" has been replaced with "BLa" as requested.

C15. Return is important for racket sports so it should add this article to support - Kilit, B., Şenel, Ö., Arslan, E., \& Can, S. (2016). Physiological responses and match characteristics in professional tennis players during a one-hour simulated tennis match. Journal of human kinetics, 51(1), 8392.

R15. Thank you for directing us to this relevant reference. We have now included the suggested reference to support the importance of including the actual return of the shuttlecock.

\section{Responses to Reviewer 2 (Highlighted in blue)}

C1. Overview: The paper investigates the reliability of a field-based intervention to assess badminton performance. The authors have two components including a 40min test (Part A) and an open-ended test to exhaustion within or around 10 minutes. Generally, I am happy with the paper, but it needs to be clearer regarding what the aim was, how this was performed, which then needs to be reflected within the results and discussion material. Specific details for each section can be found below

R1. Thank you for your review and helpful comments. Based on your comments, and those of Reviewer 1 and the Editor, we have made substantial changes to the manuscript. We believe that the revised version is improved and hope that we have addressed the shortcomings highlighted.

C2. Point 1: Is there space for pre-amble as readers unfamiliar with badminton may be put-off. i.e. a line explaining the basics?

R2. Thank you for your suggestion. The Journal requires a structured abstract of no more than 250 words with specific sections (Purpose, Methods, Results, Conclusions). We have included the following 
introductory sentences under the Purpose section as a pre-amble: "Badminton is a sport characterized by high-intensity activity interspersed with short rests requiring specific physiological fitness, skills and game tactics."

C3. Point 2: Description of the methods starts on line three and needs to be integrated within the abstract's methods section

R3. Based on the Reviewer's comments, we have made substantial changes to the abstract. We have rearranged the information which is now combined with description of the methods within the abstract. Some information has been removed to keep in line with the 250 -word limit set by the Journal. We ask for the Reviewer's understanding.

C4. Point 3: Ln 4 -5 Part A and Part B, it is not clear why there is a part $A$ and B. Please amend purpose to make this clear

R4. The protocol was designed in two-parts. Part A aimed to mimic the activity pattern typically recorded for badminton match-play and Part B to exhaust the participants and challenge their matchfitness. We have amended the study purpose to read, "This study aimed to develop, describe and establish the reliability of a novel badminton intermittent exercise protocol, to simulate singles matchplay (Part A) and assess match-fitness (Part B).”

C5. Point 4 Ln5: Does everyone know what ghosting means or is their simpler terminology that could be used?

R5. Thank you for your feedback. The term ghosting is commonly used in racket sports to simulate practicing the movements by yourself as you visualize yourself rallying with an imaginary shuttlecock. We have added the word "simulated" in parentheses next to ghosting.

C6. Point 5: It is unclear what the 72 hrs recovery is for as you say it is for test-retest reliability. Is this for test A or B? or is it between A \& B? 
R6. The entire protocol consists of 2 parts; Part A and B. The $72 \mathrm{hrs}$ recovery period is between test sessions; participants completed the entire protocol on two separate occasions. We have re-written the section and the sentence now reads, "Twelve well-trained male badminton players performed a twopart protocol, mimicking the demands and activity of match-play, on two occasions with at least 72 hours recovery." The sentences describing Part A and B come after. We hope that the clarity of the abstract is improved and apologize for any confusion.

C7. Point 6: Again, I am not sure what the comparison of results is between TEST A\&B or A1vsA2 etc?

R7. We now specify the specific section for test-retest reliability in the Results.

\section{C8. Point 7: What is the new protocol A or B?}

R8. We apologize for any confusion; the new protocol is a single protocol, consisting of a Part A and Part B, which was performed on two separate occasions.

\section{C9. Pg4: you talk about the interaction of arm work. Has anybody investigated this prior to your} work?

R9. Please could we ask the Reviewer to clarify if we have misunderstood the comment as we are genuinely uncertain as to whether the Reviewer is referring to the interaction between arm work and fatigue experienced, shot frequency or the return of shuttle. To the authors' knowledge, to date, no studies have specifically examined the interaction of arm work with any of the above. However, the importance of the return of shuttlecock is emphasized in the following article highlighted by Reviewer 1 - Kilit, B., Şenel, Ö., Arslan, E., \& Can, S. (2016). Physiological responses and match characteristics in professional tennis players during a one-hour simulated tennis match. Journal of human kinetics, 51(1), 83-92. This has also been included in the manuscript. 
C10. Pg5: Please make the purpose clearer with regards to which test is the focus of the study i.e the 10' test (B) and what it's relationship is with the 40' test (A)

R10. We apologize for the confusion. The protocol was designed in two-parts both of which were under evaluation. Part A offers a systematic protocol which mimics the activity pattern typically recorded for badminton match-play with the potential of being used for research purposes and/or self-training. Part B provides a fatiguing protocol which can be extended from Part A as a tool to assess match-fitness. We have now specified these details to clarify the aim of this study.

C11. I am not particularly keen on the experimental approach to the problem section. I feel that any relevant information from this section should be included within the procedures section.

R11. We have moved this information to the Procedures section of the Methods as requested.

C12. Pg. 6 You mention that the launcher has accuracy of $89 \%$. What happens during the test when it misses?

R12. The test continues even on instances where the launcher fails to launch accurately. This has also been included in the manuscript.

C13. Be good to see a video demo of the protocol in supplementary material if allowed or reference to it in https://figshare.com/ or similar

R13. Thank you for your suggestion. We will include a supplementary file showing a demonstration sequence of the protocol in the Journal website.

C14. Pg10,Ln 4, should read $\leq 10$ minutes. On this point I am very interested in why subjects only lasted just over 3 minutes. This seems like a limitation in the test

R14. This has been changed as requested. Thank you for your feedback. The aim was not to exhaust participants in 10 minutes but within that time frame which we did not specify. We would like to direct the Reviewer's attention to the data from top level badminton players included in the Discussion section of this manuscript. Here, the players lasted on average 5 minutes, consistent with data reported by 
Nicholas and colleagues (2000) - "Part B was $6.3 \pm 2.0 \mathrm{~min}$ for Trial 1 and $6.1 \pm 1.3 \mathrm{~min}$ for Trial 2" using a similar fatiguing protocol (Nicholas, C. W., Nuttall, F. E., \& Williams, C. (2000). The Loughborough Intermittent Shuttle Test: a field test that simulates the activity pattern of soccer. Journal of sports sciences, 18(2), 97-104.). Given the time taken to exhaustion, it is unlikely that the players stopped because of "boredom or the need to fulfil other obligations" (Atkinson, G., \& Nevill, A. (2005). Mathematical constants that vary? Medicine and Science in Sports and Exercise, 37(10), 1822; author reply 1823.). In this case, match-fitness is a more probable reason for stopping the test. This additional information has also been included in the Badminton intermittent exercise protocol - Design, Development and Procedures- Method and Discussion sections of the manuscript. Thus, it is worth highlighting that time to fatigue varies between players of different standards with physiological responses being reflective of fatigue.

C15. Pg10 You have used $p<0.050$ in the abstract but here it is 0.05 . please be consistent throughout. Also, why perform power analysis for sample size estimation and ignore it anyway. Please remove this statement

R15. We apologize for the lack of consistency and we have now replaced the phrase " $p<0.050$ " in the abstract with " $p<.05$ ". The statement relating to power analysis and sample size estimation has also been removed as requested.

C16. Sorry to keep referring to this but I would like to see clearer reference regarding TEST A \& B. When you refer to Trial 1 is that $A$ and 2 B? Please change to trial $1 \& 2$ throughout or A \& B. However, the figures suggest this is a test re-test trial for PART B. It looks like you have neglected to report on PART A. Please amend the paper to include PART A in all aspects or remove if irrelevant to the purpose.

R16. We apologise if our presentation of the information was confusing. As per our earlier comments for the abstract, this is a single protocol divided into two parts which follow directly onto the back of each other (Part A - 40 mins; Part B - until exhaustion) which was performed on two separate occasions (Trial 1 and Trial 2). The figures show only Part B which is the exhaustive/fatiguing part of the protocol. 
These figures demonstrate the test-retest (Trial 1 and 2) performance and physiological responses to Part B of the protocol. We hope this clarifies.

\section{C17. Use of b·min-1 or bpm?}

R17. We have standardised as $\mathrm{b} \cdot \mathrm{min}^{-1}$.

C18. I am not sure that the assumption that physiological measure obtained within 4 mins means the activity is similar to an activity lasting $\mathbf{4 0}$ mins with the same physiological data. Surely, a much higher intensity activity reflects different aspects of performance to the game? Also, see on pg14

R18. We apologize as we believe that this comment arises from the confusion related to Part A and B of the protocol. Physiological measures were obtained both during Part A (40 mins) and Part B (exhaustion portion) of the protocol. Mean HR is reflective of the physiological exertion across the entire duration of the test ( $\sim 3$ minutes) which is similar in duration to that of badminton match play. Blood lactate, however, is reflective of the point of exhaustion in the protocol which probably explains why it was at the upper end of that reported during badminton matches as it likely reflects concentrations at the end of games. Similarly, peak heart rate is reflective of end of game heart rate or a point of high intensity during game play. Coming on the back of the forty 1-minute stages of Part A of the protocol, the physiological measures recorded are similar to those reported in notational analyses. We agree, however, that as badminton is not a steady-state endurance sport it is difficult to use single measures to reflect all periods in the game which is why we use mean along with peak values for heart rate and RPE.

C19. Pg12 CV 13\%. Please clarify whether this is between participants or within participants. Possibly should be mentioned in stats sections of methods if not already.

R19. It represents the mean of the within participant variation. This has been included in the Statistical Analysis - Methods section. 
C20. PG12 you mention how TTE protocols lend themselves to small changes in power that produce large changes in TTE. However, I believe you suggest that there is no difference between trials, so no learning effect took place. So, what was the cause? I suspect more than 2 trials were required.

R20. The CV represents the variance within the protocol (not positive or negative) which we wanted to highlight and not differences between trials. TTE protocols may be more sensitive to this variance than time trial protocols but this does not mean that the variance is systematic or associated with learning. We did not quantify learning effects with the current protocol but do not discount the Reviewer's comment that more trials may demonstrate systematic learning. We have added this is as a suggestion/consideration for future research at the end of the Discussion section.

C21. Pg15, you have brought up some unpublished data on athletes of a higher standard. Is it possible to include this within the study and show data regarding rank etc.

R21. We cannot include all the data as these athletes completed a separate randomized trials evaluating the effects of a nutritional supplement on badminton performance. Nevertheless, within the elite players we ran a Spearman correlation between World Rankings at the time of test and time to exhaustion $\left(\mathrm{R}_{\mathrm{s}}\right.$ $=0.26, p>.05$ ) using placebo (non-supplement) data. The finding suggests that within this narrow group of elite athletes, exercise duration for Part B of the protocol is not simply reflective of greater skill level. We assume that the longer duration reflects match-fitness.

\section{C22. Pg15 Still the better players didn't get near 10mins}

R22. Agreed. As stated in our response to Comment 14, at the planning stage of the protocol the aim was to exhaust participants within 10 minutes and not make 10 minutes a target for exhaustion. A shorter exhaustion time is not a limit of the protocol and other extant protocols which mimic match-play in different sports (e.g. Soccer-Nicholas, C. W., Nuttall, F. E., \& Williams, C. (2000). The Loughborough Intermittent Shuttle Test: a field test that simulates the activity pattern of soccer. Journal of sports sciences, 18(2), 97-104.) have similar exhaustion times. What we believe is most important is the point that the protocol is able to differentiate players of different standard. This allows it is to be used for 
assessment of match-fitness or be useful to coaches/athletes in determining changes in fitness with training regimens.

\section{C23. Change "What does this article add?" to Practical implications}

R23. We are not disagreeable with the Reviewer's suggestion but the 'Instructions for Authors' on the Journal website state that this terminology should be used. Please see the website: https://www.tandfonline.com/action/authorSubmission?show=instructions\&journalCode=urqe20 We have left the heading for now and ask for the Reviewer's understanding of the instructions/constraints set by the Journal.

C24. The question here is whether your test does reflect a player's fitness or their skill level or rather their performance level. This could be suggested if you include player rank and performance in test within the results

R24. We agree that skill and fitness are different constructs and that the protocol includes elements of both. However, as stated in our response to Comment 21, within elite players there was no relationship between World Rankings and time to exhaustion suggesting that skill is not the only element at play. Yet, there was a clear difference between the elite players and those tested in this development part of the protocol. However, there are likely to be differences in both skill and fitness between elite and competitive sub-elite players. Thus, we believe that the protocol is reflective of match-fitness whilst performing an ecologically valid task. 
(a)

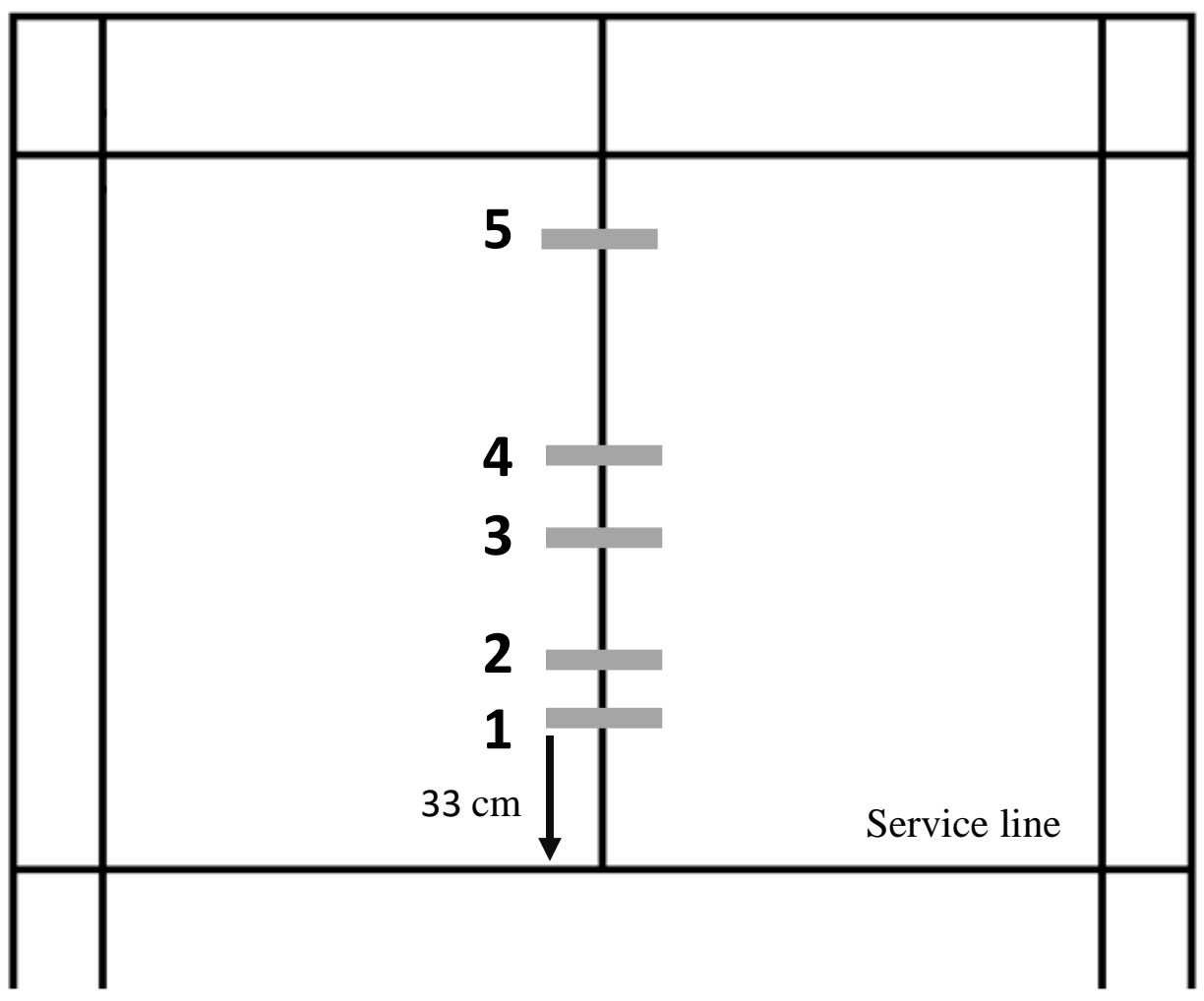

\begin{tabular}{|c|c|}
\hline Position & $\begin{array}{c}\text { Distance from } \\
\text { service line (cm)* }\end{array}$ \\
\hline 1 & 33 \\
\hline 2 & 38 \\
\hline 3 & 110 \\
\hline 4 & 113 \\
\hline 5 & 371 \\
\hline
\end{tabular}

* Measured from the left wheel of the machine as shown in Position 1

(b) Opposite side of court

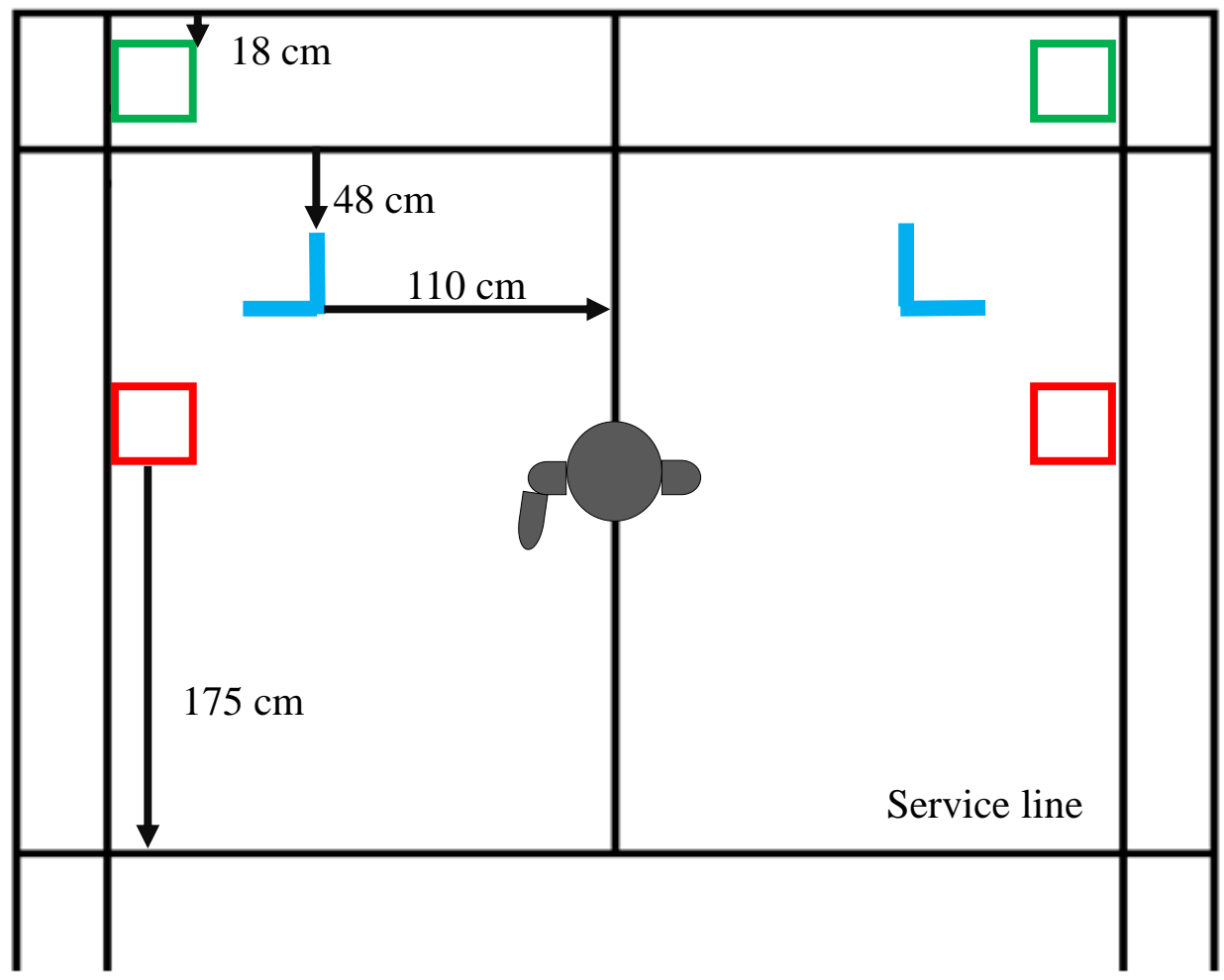

$40 \mathrm{~cm}$

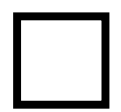

$40 \mathrm{~cm}$

(c)

\begin{tabular}{|c|c|c|}
\hline Marker & Dummy shot type(s) & Instruction \\
\hline Green box & Clear, Drop & One foot in the green box \\
\hline Blue "L" & Smash & One foot within the blue "L" \\
\hline Red box & Block, Drive & Racket head above the red box \\
\hline Service line & Net, Lift, Push & One foot on the service line \\
\hline
\end{tabular}



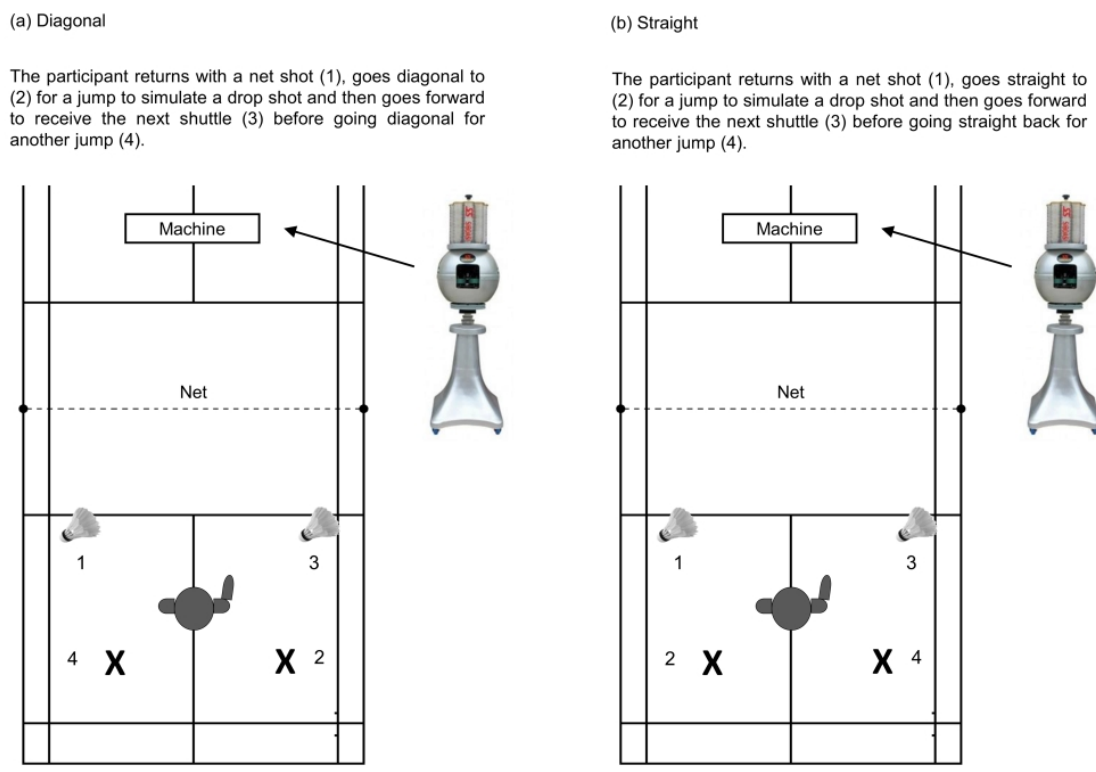
(2) for a jump to simulate a drop shot and then goes forward another jump (4).

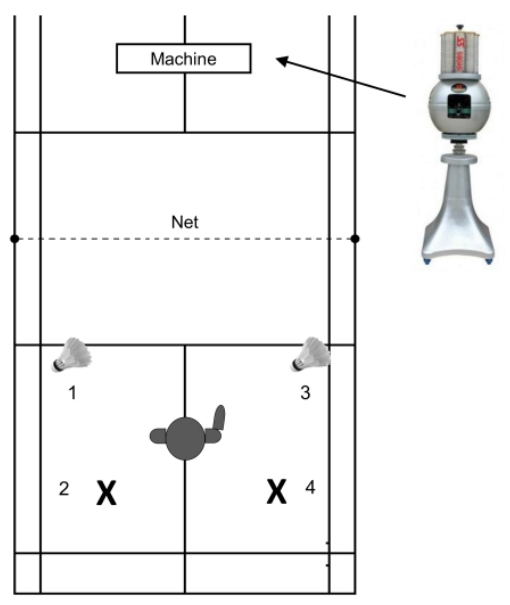

Overview of the experimental set-up illustrating a sample sequence of the protocol. $225 \times 160 \mathrm{~mm}(600 \times 600 \mathrm{DPI})$ 
(a)

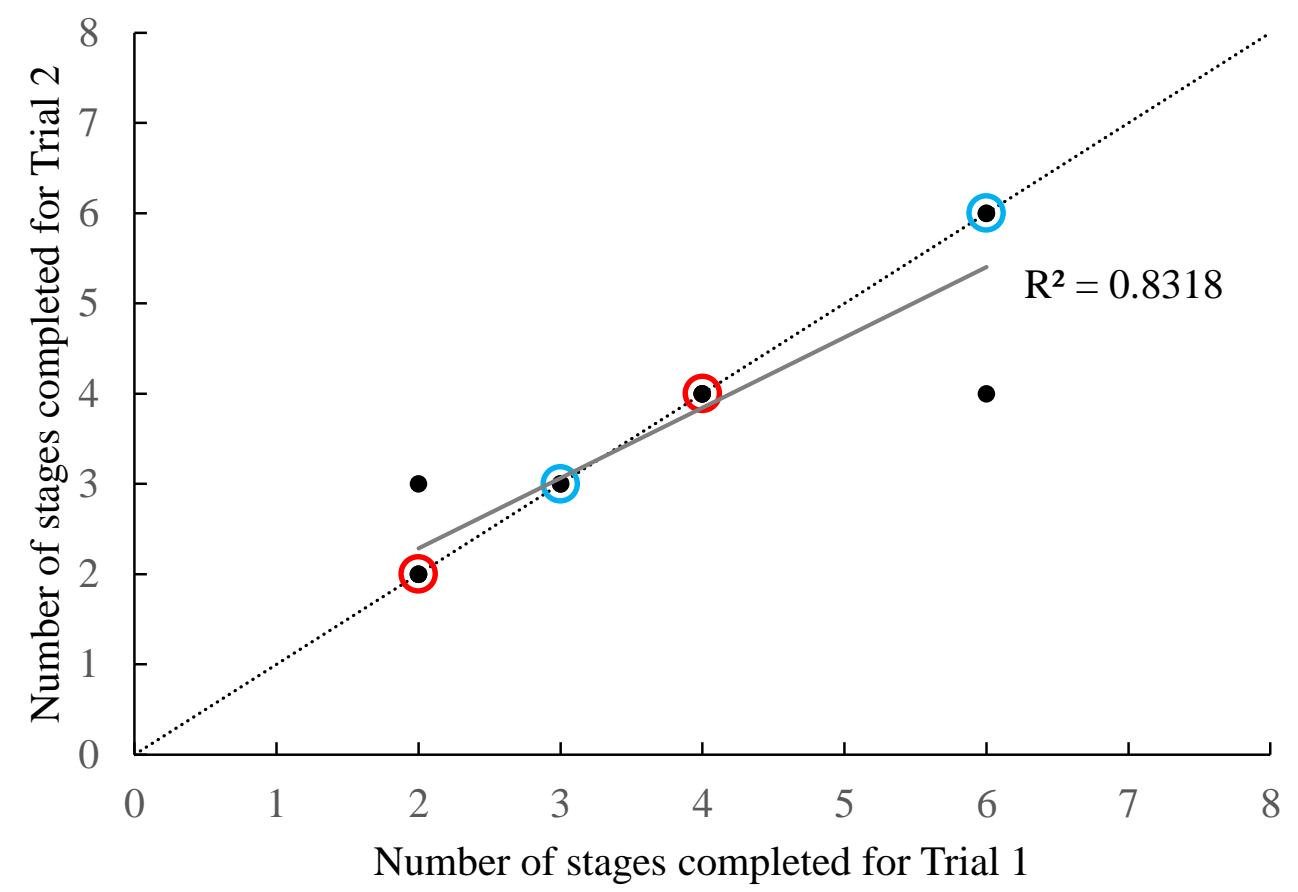

(b)

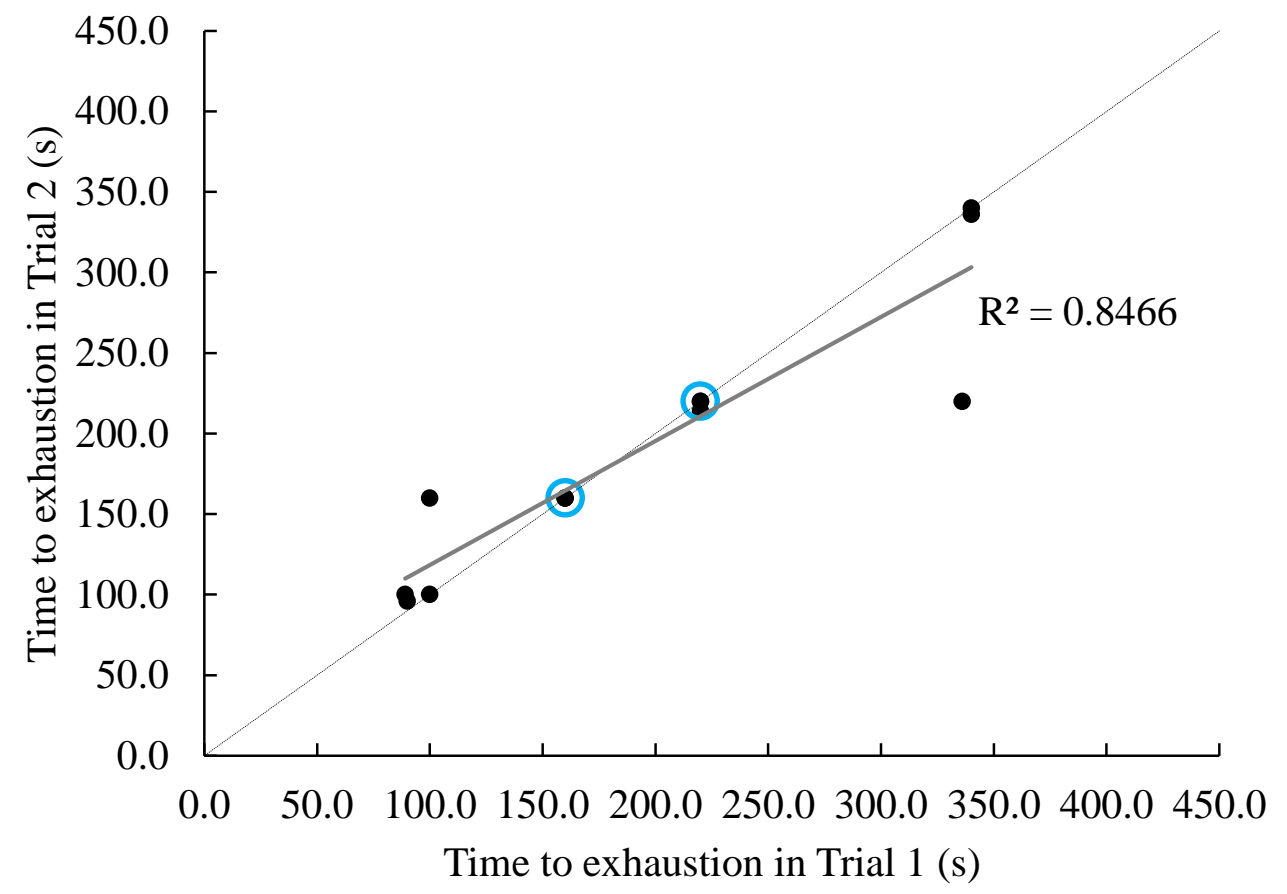


(a)

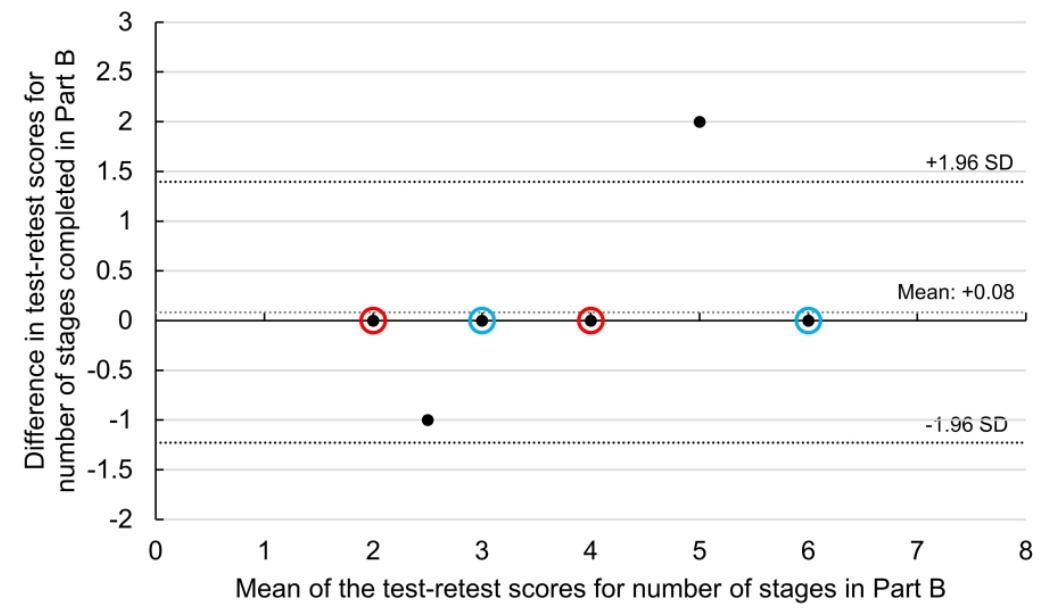

(b)

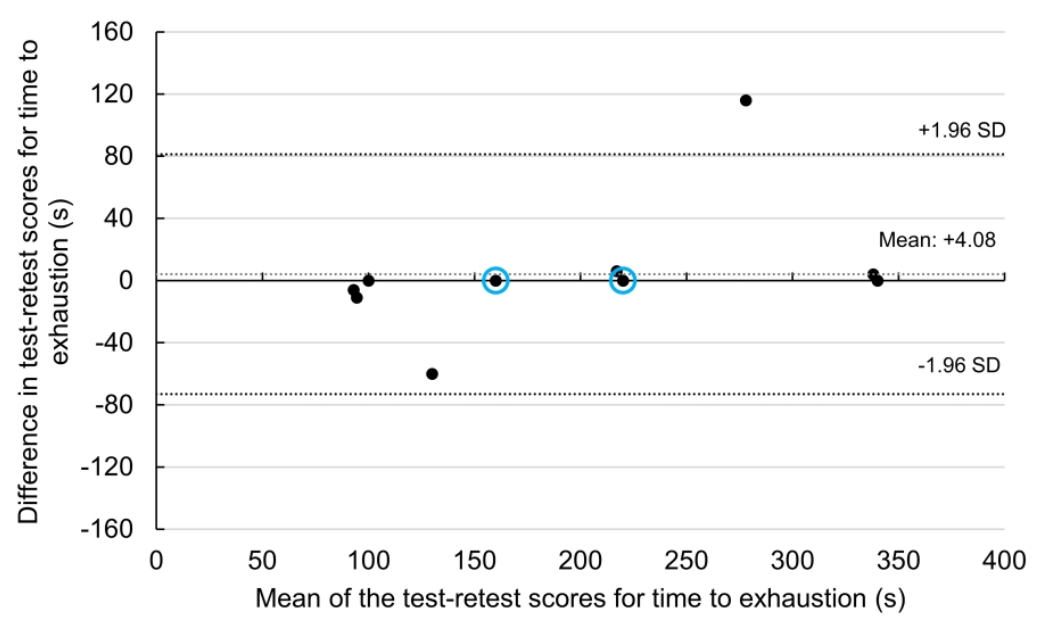

Bland-Altman plot for test-retest scores for (a) number of stages completed and (b) time to exhaustion ( $\mathrm{n}=$ 12). Blue and red circles indicate a duplicate of two and three participants respectively.

$$
190 \times 225 \mathrm{~mm}(600 \times 600 \mathrm{DPI})
$$


(a)

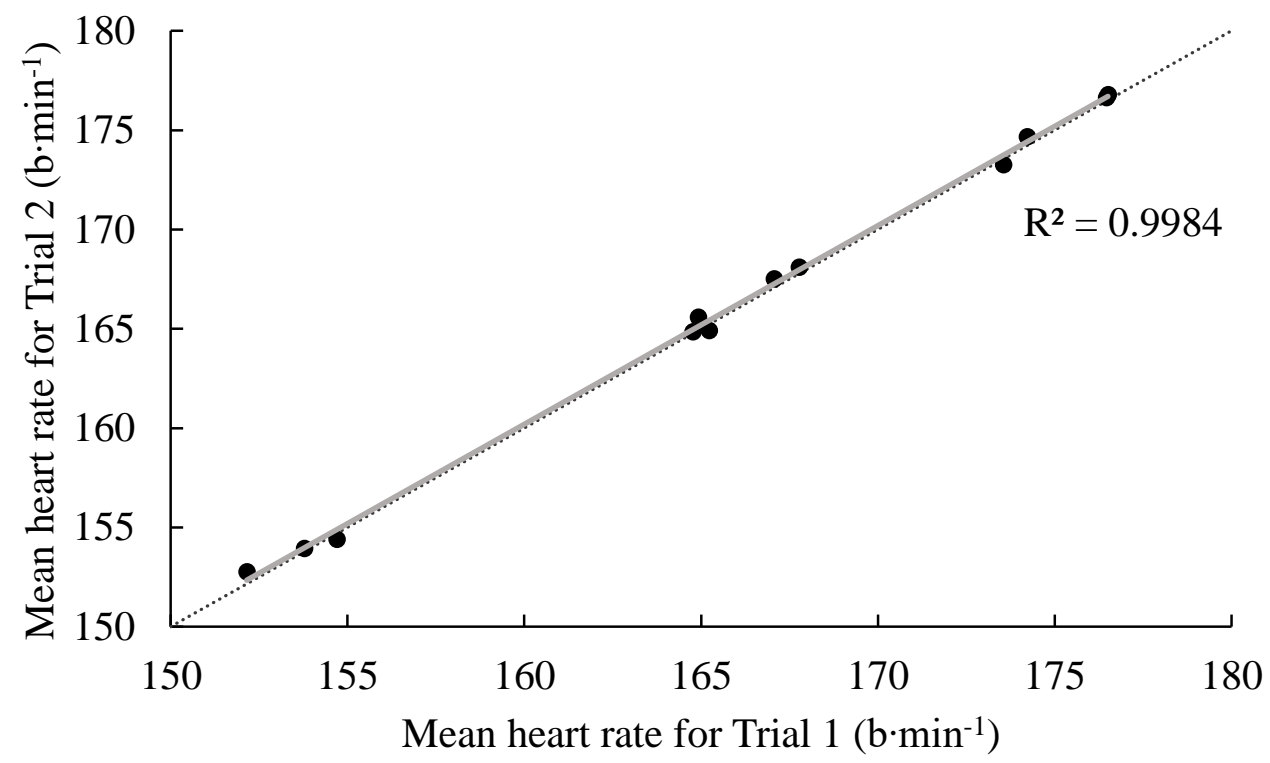

(b)

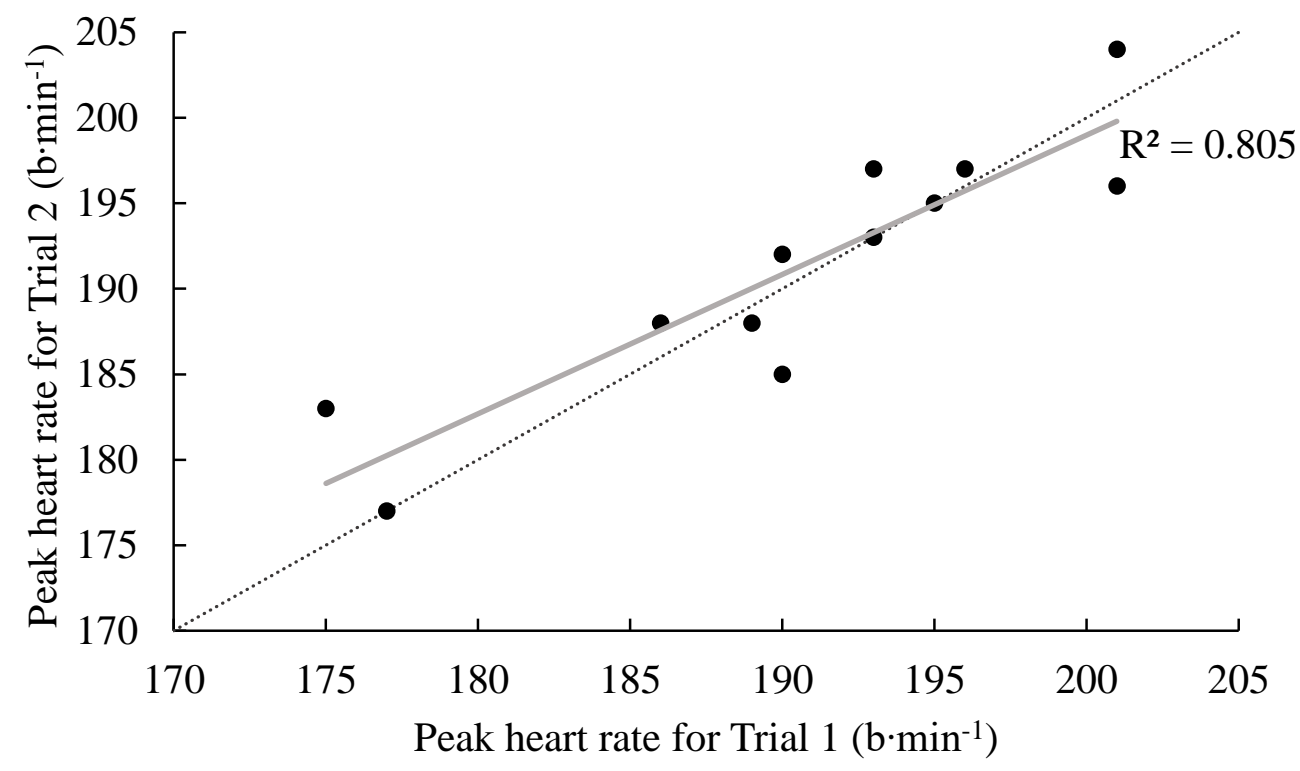

(c)

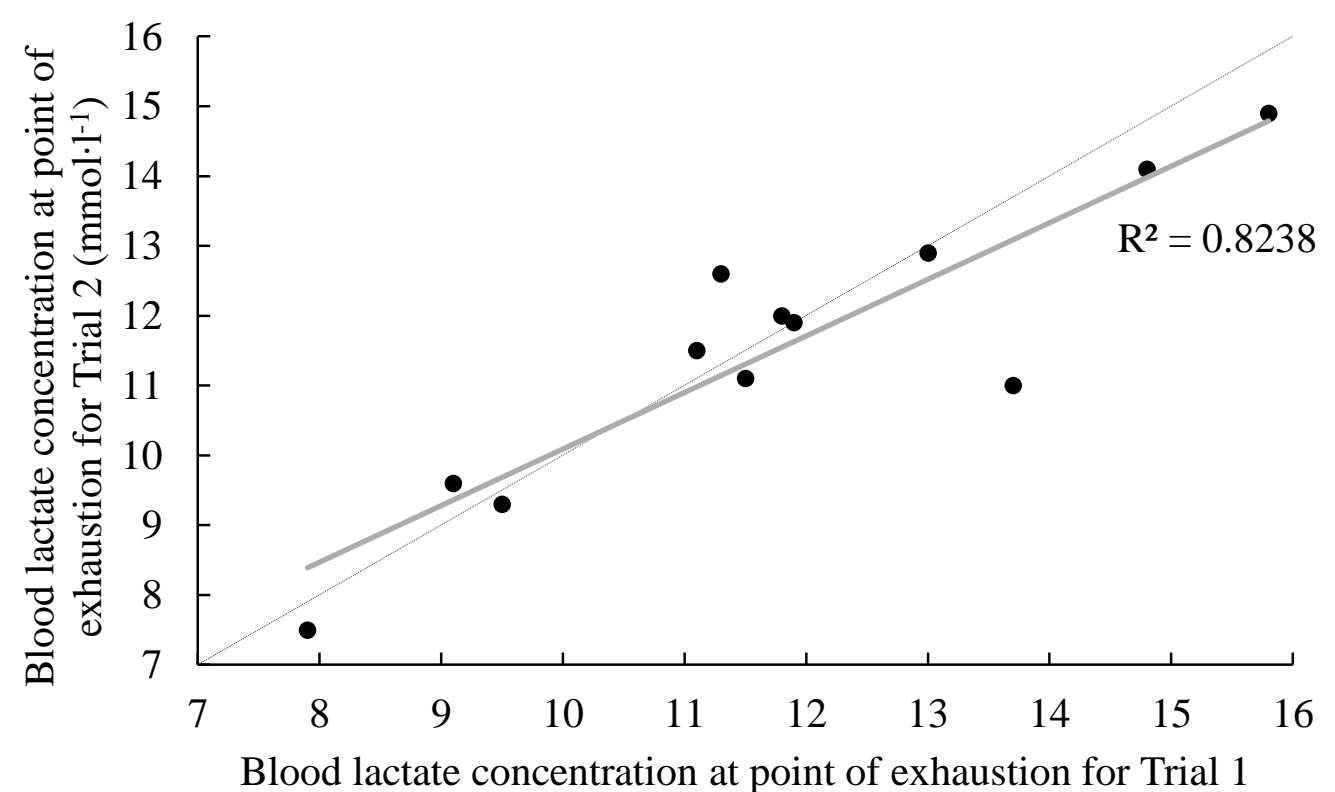

$\left(\mathrm{mmol} \cdot \mathrm{l}^{-1}\right)$ 
Table 1 Shot type distribution.

\begin{tabular}{lcccccccc}
\hline & \multicolumn{8}{c}{ Shot Type } \\
& Clear & Smash & Drop & Drive & Lift & Net & Push & Block \\
Percentage & 12.3 & 16.4 & 7.8 & 9.4 & 14.2 & 16.1 & 15.0 & 8.8 \\
& & & & & & & & \\
\hline
\end{tabular}


Table 2. Mean \pm SD, TEM, LOA, ICC with $95 \%$ CI, CV and significance values.

\begin{tabular}{|c|c|c|c|c|c|c|c|}
\hline \multirow[b]{3}{*}{ Performance } & \multicolumn{2}{|c|}{ Mean \pm SD } & \multirow[t]{2}{*}{ TEM } & \multirow{2}{*}{$\begin{array}{c}95 \% \text { LOA } \\
\text { (lower, upper) }\end{array}$} & \multirow[t]{2}{*}{$\mathrm{ICC}_{2,1}(95 \% \mathrm{CI})$} & \multirow{2}{*}{$\begin{array}{l}\mathrm{CV} \\
(\%)\end{array}$} & \multirow[t]{2}{*}{$p^{*}$} \\
\hline & Trial 1 & Trial 2 & & & & & \\
\hline & & & & & & & \\
\hline Stages completed & $4 \pm 2$ & $4 \pm 1$ & 0.5 & $-1.23,1.39$ & $0.907(0.712-0.972)$ & 13.0 & 0.674 \\
\hline Time to exhaustion (s) & $198 \pm 98$ & $194 \pm 82$ & 27.9 & $-73.11,81.28$ & $0.912(0.725-0.974)$ & 14.7 & 0.726 \\
\hline \multicolumn{8}{|l|}{ Heart rate } \\
\hline $\mathrm{HR}_{\text {Mean }}\left(\mathrm{b} \cdot \mathrm{min}^{-1}\right)$ & $166 \pm 9$ & $166 \pm 9$ & 0.5 & $-1.73,1.23$ & $0.996(0.987-0.999)$ & 0.3 & 0.275 \\
\hline $\mathrm{HR}_{\text {Peak }}\left(\mathrm{b} \cdot \mathrm{min}^{-1}\right)$ & $191 \pm 8$ & $191 \pm 7$ & 2.5 & $-7.80,6.30$ & $0.897(0.690-0.969)$ & 1.4 & 0.485 \\
\hline \multicolumn{8}{|l|}{ Blood lactate } \\
\hline $\mathrm{BLa}_{\text {End }}\left(\mathrm{mmol} \cdot \mathrm{l}^{-1}\right)$ & $11.8 \pm 2.3$ & $11.5 \pm 2.1$ & 0.7 & $-1.65,2.15$ & $0.903(0.710-0.971)$ & 5.8 & 0.391 \\
\hline \multicolumn{8}{|l|}{ Perceived exertion } \\
\hline $\mathrm{RPE}_{\text {PartA }}$ & $13 \pm 3$ & $12 \pm 2$ & 0.7 & $-2.32,1.82$ & $0.913(0.736-0.974)$ & 5.8 & 0.429 \\
\hline $\mathrm{RPE}_{\text {End }}$ & $19 \pm 1$ & $20 \pm 1$ & 0.7 & $-2.54,1.37$ & $0.304(-0.179-0.713)$ & 3.8 & 0.067 \\
\hline
\end{tabular}

Note. BLa: blood lactate, CI: confidence interval, CV: coefficient of variation, HR: heart rate, ICC: intraclass correlation coefficient, LOA: limits of agreement, RPE: rating of perceived exertion, TEM: typical error of measurement, *: paired $t$-test between Trial 1 and 2. 
Full set of drill sequences indicating the type of shots and machine positions

\begin{tabular}{|c|c|c|c|c|c|}
\hline Drill & Shot & Dummy & Specific Instructions & Variation(s) & Machine Position \\
\hline \multicolumn{6}{|c|}{ Part A } \\
\hline 1 & Net & Clear & 1 foot in blue "L" & \multirow{10}{*}{ Diagonal, straight } & \multirow{3}{*}{5} \\
\hline 2 & Net & Drop & 1 foot in blue "L" & & \\
\hline 3 & Lift & Drive & Racket above red box & & \\
\hline 4 & Drive & Net & 1 foot on service line & & 4 \\
\hline 5 & Smash & Net & 1 foot on service line & & \multirow{2}{*}{3} \\
\hline 6 & Smash & Push & 1 foot on service line & & \\
\hline 7 & Lift & Lift & 1 foot on service line & & \multirow{2}{*}{2} \\
\hline 8 & Block & Clear & 1 foot in green box & & \\
\hline 9 & Clear & Block & Racket above red box & & \multirow{2}{*}{1} \\
\hline 10 & Drop & Push & 1 foot on service line & & \\
\hline \multicolumn{6}{|c|}{ Part B } \\
\hline 11 & Smash & Push & 1 foot on service line & Straight & 1 \\
\hline
\end{tabular}

*Shot: participant receives the shuttle from the machine and is required to return the shuttle using the specified shot type; Dummy: participant is required to perform a ghosting action of the shot specified; Machine position and markers: refer to Figure 1. 